\title{
High throughput 16SrRNA gene sequencing reveals the correlation between Propionibacterium acnes and sarcoidosis
}

\author{
Meng-Meng Zhao ${ }^{\dagger}$, Shan-Shan Du ${ }^{\dagger}$, Qiu-Hong Li ${ }^{\dagger}$, Tao Chen $^{\dagger}$, Hui Qiu, Qin Wu, Shan-Shan Chen, Ying Zhou, \\ Yuan Zhang, Yang Hu, Yi-Liang Su, Li Shen, Fen Zhang, Dong Weng ${ }^{*}$ and Hui-Ping Li ${ }^{*}$
}

\begin{abstract}
Objective: This study aims to use high throughput 16SrRNA gene sequencing to examine the bacterial profile of lymph node biopsy samples of patients with sarcoidosis and to further verify the association between Propionibacterium acnes (P. acnes) and sarcoidosis.

Methods: A total of 36 mediastinal lymph node biopsy specimens were collected from 17 cases of sarcoidosis, 8 tuberculosis (TB group), and 11 non-infectious lung diseases (control group). The V4 region of the bacterial 16SrRNA gene in the specimens was amplified and sequenced using the high throughput sequencing platform MiSeq, and bacterial profile was established. The data analysis software QIIME and Metastats were used to compare bacterial relative abundance in the three patient groups.

Results: Overall, 545 genera were identified; 38 showed significantly lower and 29 had significantly higher relative abundance in the sarcoidosis group than in the TB and control groups $(P<0.01)$. P. acnes 16 SrRNA was exclusively found in all the 17 samples of the sarcoidosis group, whereas was not detected in the TB and control groups. The relative abundance of $P$. acnes in the sarcoidosis group $(0.16 \% \pm 0.11 \%)$ was significantly higher than that in the TB (Metastats analysis: $P=0.0010, q=0.0044$ ) and control groups (Metastats analysis: $P=0.0010, q=0.0038$ ). The relative abundance of P. granulosum was only $0.0022 \% \pm 0.0044 \%$ in the sarcoidosis group. P. granulosum 16SrRNA was not detected in the other two groups.
\end{abstract}

Conclusion: High throughput 16SrRNA gene sequencing appears to be a useful tool to investigate the bacterial profile of sarcoidosis specimens. The results suggest that P. acnes may be involved in sarcoidosis development.

Keywords: High throughput gene sequencing, Propionibacteria, Sarcoidosis, 16SrRNA

\section{Background}

Sarcoidosis is a systemic granulomatous disease and often involves the respiratory system. The disease frequently affects middle-aged adults, in particular women aged $40-50$ years. The histopathology of sarcoidosis is characterized by non-caseous necrotizing granulomas, and the clinical presentation of the disease is very diverse [1]. The exact etiology and pathogenesis of sarcoidosis remain unclear although several factors, such as infection [2], genetic defects [3], immune dysfunction

\footnotetext{
* Correspondence: cruise00@gmail.com; liw2013@126.com

${ }^{\dagger}$ Equal contributors

Department of Respiratory Medicine, Shanghai Pulmonary Hospital, Tongji

University, School of Medicine, 507 Zheng Min Road, Shanghai 200433, China
}

[4], and exposure to environmental pollutants [5], have been indicated to contribute to sarcoidosis development.

Previous reports studying the association of infection and sarcoidosis mainly focus on Mycobacterium tuberculosis (MTB) and propionibacteria, and show controversial results. Eishi et al. [6] used real-time quantitative RT-PCR to analyze bacterial DNA in the lymph node biopsy specimens of patients with sarcoidosis, TB, or other lung diseases and suggested that propionibacteria was more likely than mycobacterium to be an etiological factor of sarcoidosis. Contrarily, other reports indicate a correlation of MTB and sarcoidosis. Oswald et al. [7] found that the CD4+ and/or CD8+ T cells isolated from the bronchoalveolar lavage fluid of patients with 
sarcoidosis were more likely than the $\mathrm{T}$ cells from the control patients to be stimulated by mycobacterial ESAT-6 protein, whereas the response to P. acnes proteins was similar between the $\mathrm{T}$ cells of the sarcoidosis group and the $\mathrm{T}$ cells of the control group. Our previous studies used PCR to determine the copy number of mycobacteria and propionibacteria in the paraffinembedded lymph node biopsy specimens of patients with sarcoidosis, TB, or other lung diseases and indicated that MTB might not be associated with sarcoidosis [8] but propionibacteria could be [9].

Most of the previous studies investigated only a few bacterial species. A full bacterial profile of the tissue specimens of patients with sarcoidosis is still lacking. The current study is filling this knowledge gap. In the current study, we aim to conduct high throughput 16SrRNA gene sequencing to investigate the bacterial profile of mediastinal lymph node specimens. The 16SrRNA gene is present in the genome of bacteria, chlamydia, rickettsia, mycoplasma, spirochaeta, and actinomycetes, but absent in eukaryotic organisms, viruses, and fungus. The sequence of $\mathrm{V} 4$ region of the bacterial 16SrRNA gene, which varies greatly in different bacterial species, is often considered as a unique signature for a bacterial species and thus commonly used for bacterial taxonomic classification [10-12]. The V4 region is flanked by evolutionary conserved regions. In the current study, the conserved regions were used to design PCR primers. The V4 region was then amplified by PCR with the primers and then sequenced. The sequences were analyzed and compared to the 16SrRNA gene sequence database, GreenGene database, by bioinformatics approaches to identify the bacterial taxonomic annotation $[11,13]$. In the current study, we are to use bacterial 16SrRNA gene sequencing to characterize the bacterial profile of the tissue specimens of patients with sarcoidosis and identify the potential pathogenic bacteria that may correlate with the disease.

\section{Methods}

\section{Tissue specimens}

The protocol for collecting and handling patients' biopsy specimens has been approved by the Institutional Review Board of Shanghai Pulmonary Hospital and Tongji University School of Medicine (Approval No: 2011-FK10). Biopsy specimens from the mediastinal lymph node of 36 patients, who were treated in Shanghai Pulmonary Hospital between August 2014 and August 2015, were collected and analyzed. All the 36 patients, including 17 cases of sarcoidosis, 8 cases of TB, and 11 cases of control, were inpatients of Shanghai Pulmonary Hospital. The 8 patients with TB were newly diagnosed based on positive MTB in their sputum. The 11 controls included 6 cases of nonspecific lymphadenitis and 5 cases of mediastinal lymph node metastasis of lung cancer. The clinical data of the patients are displayed in Table 1. All the participating patients signed the informed consent.

\section{Diagnostic criteria}

The diagnostic criteria for sarcoidosis followed the 1999 guidelines of ATS/ERS/WASOG [1]. The histopathology of lymph node biopsy of the patients with sarcoidosis was characterized by non-caseous necrotizing granulomas and showed negative TB, fungus and/or parasitic infection, tumor, and vasculitis. Additionally, to further confirm sarcoidosis, smear-negative TB was excluded based on a negative TB-polymerase chain reaction (PCR) [8] and the comprehensive differential diagnosis scoring system for sarcoidosis and atypical TB [14]. All the 17 patients with sarcoidosis were followed up for 1 year.

Patients with TB were diagnosed according to the 2013 tuberculosis diagnosis and treatment guidelines recommended by the China Medical Association Tuberculosis Society [15]. The diagnosis of TB was based on acid-fast bacilli smear-positive sputum, positive Mycobacterium tuberculosis (M. TB) in sputum culture, positive $\mathrm{M}$. TB in bronchopulmonary lavage fluid, or positive staining for acid-fast bacilli in biopsy specimens. All the patients with TB underwent anti-TB therapy and cured. The lymph node biopsy samples of lymphadenitis and lung cancer metastasis were provided by thoracic surgeons of Shanghai Pulmonary Hospital, and the diagnosis was confirmed.

\section{Gene sequencing of 16SrRNA}

Because the sequence of $\mathrm{V} 4$ region of the bacterial 16SrRNA gene is a unique signature of a bacterial species, the sequence can be used to identify the specific bacterial taxonomic position [10]. The V4 region is flanked by evolutionary conserved regions, which were used to design PCR primers. The V4 region was then amplified by PCR with the primers and then sequenced. Genomic DNA was extracted from the tissue specimens using the TIANquick FFPE DNA Kit (TIANGEN, China) according to the instruction of the kit. The genomic DNA was then used as the template in the PCR to amplify the 16SrRNA gene V4 region (nucleotide position from 515 to 806). The primer sequences are: 5'GTGCCAGCMGCCGCGGTA-3' (forward primer) and 5'-GGACTACHVGGGTWTCTAAT-3' (reverse primer). $\mathrm{M}$ stands for nucleotide $\mathrm{A}$ or $\mathrm{C}$; $\mathrm{H}$ stands for nucleotide $\mathrm{A}, \mathrm{C}$, or $\mathrm{T}$; V stands for nucleotide $\mathrm{A}, \mathrm{C}$, or $\mathrm{G}$; W stands for nucleotide A or T [10]. Phusion ${ }^{\circ}$ High-Fidelity PCR Master Mix (New England Biolabs, USA) was used for PCR reaction. The PCR reaction condition was: initial denaturation at $98{ }^{\circ} \mathrm{C}$ for $1 \mathrm{~min} ; 30$ cycles of denaturation at $98{ }^{\circ} \mathrm{C}$ for $10 \mathrm{~s}$, annealing at $50{ }^{\circ} \mathrm{C}$ for $10 \mathrm{~s}$, extension at $72{ }^{\circ} \mathrm{C}$ for $60 \mathrm{~s}$; final extension at $72{ }^{\circ} \mathrm{C}$ for 
Table 1 Patients' clinical data

\begin{tabular}{|c|c|c|c|c|}
\hline & $\begin{array}{l}\text { Sarcoidosis } \\
N=17\end{array}$ & $\begin{array}{l}\text { Control disease } \\
N=11\end{array}$ & $\begin{array}{l}\text { Tuberculosis } \\
N=8\end{array}$ & $P$ Value \\
\hline Age, mean \pm SD (years) & $53.12 \pm 7.41$ & $53.55 \pm 12.75$ & $45.13 \pm 20.73$ & 0.3013 \\
\hline Male/female ratio & $3 / 14$ & $6 / 5$ & $6 / 2$ & N/A \\
\hline CXR stage: $0 / I / I / I I I / I V$ number of patients (n) & $0 / 10 / 4 / 3 / 0$ & N/A & N/A & N/A \\
\hline Tuberculosis examination negative/positive ( $\mathrm{n}$ ) & $17 / 0$ & N/A & $0 / 8$ & N/A \\
\hline $\mathrm{BMI}\left(\mathrm{kg} / \mathrm{m}^{2}\right)$ & $24.74 \pm 2.45$ & $22.75 \pm 3.87$ & $22.87 \pm 2.34$ & 0.1564 \\
\hline SACE (IU/L) & $58.47 \pm 26.86(n=15)$ & N/A & N/A & N/A \\
\hline $\operatorname{ESR}(\mathrm{mm} / \mathrm{h})$ & $24.13 \pm 15.82(n=16)$ & $31.20 \pm 19.81(n=6)$ & $34.57 \pm 39.43(n=7)$ & 0.5996 \\
\hline 24-h urinary calcium & $5.70 \pm 2.11(n=12)$ & N/A & N/A & N/A \\
\hline CD3 (\%) & $66.56 \pm 8.07(n=14)$ & N/A & $58.67 \pm 15.58(n=7)$ & 0.1380 \\
\hline CD4 (\%) & 34. $01 \pm 10.15(n=14)$ & N/A & $35.66 \pm 13.03(n=7)$ & 0.7526 \\
\hline CD8 (\%) & $24.87 \pm 10.40(n=14)$ & N/A & $18.99 \pm 4.51(n=7)$ & 0.1730 \\
\hline $\lg G(n g / L)$ & $12.36 \pm 2.73(n=10)$ & N/A & $14.67 \pm 7.20$ & 0.3607 \\
\hline $\lg A(n g / L)$ & $1.96 \pm 0.56(n=10)$ & N/A & $2.73 \pm 1.12$ & 0.0734 \\
\hline $\lg M(n g / L)$ & $1.47 \pm 0.77(n=10)$ & N/A & $1.19 \pm 0.73$ & 0.4324 \\
\hline C3 (ng/L) & $1.25 \pm 0.20(n=10)$ & N/A & $1.20 \pm 0.29$ & 0.6584 \\
\hline C4 (ng/L) & $0.31 \pm 0.072(n=10)$ & N/A & $0.34 \pm 0.094$ & 0.4571 \\
\hline
\end{tabular}

5 min. The PCR products were electrophoresed, collected from the gel using the GeneJET Gel Extraction Kit (Thermo Scientific, USA). The purified PCR products were used to construct a DNA sequencing library using the the NEB Next ${ }^{\circ}$ Ultra $^{\text {TM }}$ DNA Library Prep Kit from Illumina (San Diego, CA, USA) according to the manufacturer's instruction. The quality of the DNA library was examined using the Quibit ${ }^{\circ}$ 3.0 Fluorometer 2.0 (Thermo Fisher Scientific, USA) and Agilent Bioanalyzer 2100 system. The DNA library was then sequenced on the Illumina MiSeq platform (CA, USA). The collected raw sequencing data were processed according to the following steps: 1) The sequences of Barcode and primers were first removed from the raw sequencing data. 2) The sequencing data were then analyzed using the software FLASH version 1.2.7 (http://ccb.jhu.edu/ software/FLASH/)[16]. For each sample, the sequencing fragments were aligned and connected to form raw sequencing tags. 3) The raw sequencing tags with low quality sequencing data were removed. 4) The final sequencing tags were compared against the Genomes OnLine Database (Gold) database (http://drive5.com/ uchime/uchime_download.html) using the UCHIME Algorithm (http://www.drive5.com/usearch/manual/uchime_algo.html) [17] to identify chimeric sequences. The chimeric sequences were removed from the final sequencing tags [18]. The resulting sequences were the effective sequencing tags and used for bacterial taxonomic annotation.

\section{Sequence analysis}

The identified 16SrRNA sequences (the effective sequencing tags) were annotated using the Uparse software package v7.0.1001 (http://drive5.com/uparse/) [19]. The annotation procedure includes the following steps: 1) Sequences that share $\geq 97 \%$ identity were considered at the same taxonomic position (belong to the same bacterial species) and assigned to one operational taxonomic unit (OTU). 2) The Uparse software automatically selected representative sequences when constructing OTUs. 3) The representative sequences were used to annotate the OTUs. OTUs were annotated with taxonomic information using the 16SrRNA gene sequence database, GreenGene database, (http://greengenes.lbl.gov/cgi-bin/nph-in dex.cgi) [20] and the RDP Classifier (Version 2.2, http:// sourceforge.net/projects/rdp-classifier/) [21]. The sequences were annotated with taxonomic Kingdom, Phylum, Class, Order, Family, Genus, and Species. The relative abundance of each bacterial species in a sample was also determined by the software.

\section{Statistical analysis}

To estimate if the identified 16SrRNA sequences could include all the bacteria in the samples, we conducted alpha diversity analysis. The alpha diversity was analyzed using the Quantitative Insights into Microbial Ecology (QIIME) software (Version 1.7.0) [22]. To evaluate alpha diversity, we calculated Chao index representing bacterial species abundance, number of observed species, and 
Shannon index representing bacterial community diversity. Rarefaction curves were plotted using the three parameters as vertical axes and the number of identified sequences as horizontal axes. Chao index, number of observed species, and Shannon index increase as the number of identified sequences increases in a sample, which indicates that unique bacterial species are increasingly identified in a sample. Saturation of the rarefaction curves indicate that the number of unique bacterial species does not increase as the number of identified sequences increases, which suggests that the identified 16SrRNA sequences may cover all the bacteria in the sample.

Bacterial relative abundance was determined to identify the most predominant bacterial species, which represent the bacterial profile in the samples. The Quantitative Insights into Microbial Ecology (QIIME) software (Version 1.7.0) and Uparse software package v7.0.1001 (http:// drive5.com/uparse/) were used to analyze bacterial abundance. The Metastats software (http://metastats.cbcb.umd.edu/) was used to compare the difference in bacterial abundance between different groups. The Metastats software is specifically designed to compare bacterial abundance by quantitatively analyzing the 16SrRNA gene sequences [23].

Intra-group comparison was performed to evaluate the differences in the bacterial profile of samples in the same patient group. Inter-group comparison was performed to estimate the difference in the bacterial profile of the sarcoidosis, control, and TB patient groups. Because bacterial profile is expected to vary considerably in different individuals, evaluation of inter- and intra-group difference may reveal the unique characteristics of bacterial profile in sarcoidosis, control, and TB patient groups. For inter-and intra-group comparison of bacterial profile, R software (version 3.1.3) was used for nonparametric multi-response permutations procedure (MRPP) [24] and the analysis of similarity (Anosim) [25]. An A value (calculated from the MRPP) $>0$ represented inter-group difference $>$ intra-group difference; $\mathrm{A}<0$ represented inter-group difference $<$ intra-group difference, $P<0.01$ was considered statistically significant. An $R$ value (calculated from the Anosim) $>0$ represented inter-group difference $>$ intra-group difference; $R<0$ represented inter-group difference $<$ intra-group difference; $P<0.01$ was considered statistically significant. A $P$ value was first calculated for inter-group difference in relative abundance. The $P$ value was then adjusted to $\mathrm{q}$ value using an algorithm established by Storey and Tibshirani [26]. Based on both the $P$ and q values, Taxonomic units showing significantly different relative abundance in the three groups were identified. When both $P<0.01$ and $\mathrm{q}$ $<0.01$, the differences were considered statistically significant. The software Graphpad prism 5 was used to prepare the curves of bacterial relative abundance. The software SPSS 20.0 was used for chi-square analysis of the inter-group difference in bacterial positive rate; $P<0.01$ was considered statistically significant.

\section{Results \\ Patients' clinical data}

Patients in the sarcoidosis, TB, and control groups showed similar age, body mass index, and erythrocyte sedimentation rate (Table 1$)$. The majority of patients $(n=10)$ with sarcoidosis were at chest X-ray stage I. The percentages of specific $\mathrm{T}$ cell populations, including $\mathrm{CD} 3, \mathrm{CD} 4$, and $\mathrm{CD} 8$, were similar in the sarcoidosis and TB groups. So were the levels of IgG, IgA, IgM, C3, and C4 (Table 1).

\section{Sequence data and OTUs}

Sequencing data are presented in Additional file 1: Table S1. A total of 8748 unique OTUs were constructed by the Uparse software, and an average of 1768 unique OTUs in each sample. The sequences of all the 8748 unique OTUs are displayed in Additional file 2: Table S2. The OTUs were annotated at phylum, class, order, family, genus, and species taxonomic levels using the RDP Classifier Version 2.2 (http://sourceforge.net/projects/rdp-classifier/) and the 16SrRNA sequence database, GreenGene database (http://greengenes.lbl.gov/cgi-bin/nph-index.cgi). The annotations of all the 8748 are presented in Additional file 3: Table S3. At the taxonomic genus level, a total of 545 unique bacterial genera were identified in all the samples. The relative abundances of the 545 bacterial genera in each sample are presented in Additional file 4: Table S4.

At the taxonomic genus level, the OTUs were assigned to 545 genera based on the Greengenes database (Additional file 4: Table S4). In the sarcoidosis group, 478 unique bacterial genera were identified; the top 10 most abundant genera were: Shewanella, Pseudomonas, Acinetobacter, Lactobacillus, Prochlorococcus, Bifidobacterium, Escherichia, Halomonas, Pediococcus, and Rhodococcus (Table 2). The control group showed 470 unique bacterial genera, including Bacteroides, J2-29, Prevotella, Methylobacterium, Oscillospira, [Prevotella], Roseburia, Ruminococcus, Clostridium, and Helicobacter as the top ten most abundant bacterial genera (Table 2). The TB group contained 489 unique bacterial genera, and the most abundant ones were Bacteroides, J2-29, [Prevotella], Methylobacterium, Prevotella, Clostridium, Methyloversatilis, Succinivibrio, Sutterella, and Salinispora (Table 2). All of the most abundant bacterial genera in the three patient groups belonged to the four bacterial Phylum: Proteobacteria, Firmicutes, Bacteroidetes, and Fusobacteria. 
Table 2 The top ten most abundant bacterial genera of the three patient groups

\begin{tabular}{|c|c|c|c|c|c|c|}
\hline & Sarcoidosis & & Control disease & & Tuberculosis & \\
\hline & Bacteria & Abundance & Bacteria & Abundance & Bacteria & Abundance \\
\hline 1 & Shewanella & $7.33 \pm 4.60$ & Bacteroides & $13.13 \pm 2.72$ & Bacteroides & $13.32 \pm 3.51$ \\
\hline 2 & Pseudomonas & $4.32 \pm 2.75$ & $J 2-29$ & $5.28 \pm 3.82$ & $J 2-29$ & $8.91 \pm 2.15$ \\
\hline 3 & Acinetobacter & $3.31 \pm 4.46$ & Prevotella & $5.16 \pm 2.63$ & [Prevotella] & $3.25 \pm 0.89$ \\
\hline 4 & Lactobacillus & $3.06 \pm 3.98$ & Methylobacterium & $4.11 \pm 3.95$ & Methylobacterium & $2.96 \pm 2.85$ \\
\hline 5 & Prochlorococcus & $2.24 \pm 2.83$ & Oscillospira & $3.36 \pm 1.92$ & Prevotella & $2.75 \pm 1.29$ \\
\hline 6 & Bifidobacterium & $1.89 \pm 7.05$ & [Prevotella] & $3.16 \pm 1.02$ & Clostridium & $2.60 \pm 0.25$ \\
\hline 7 & Escherichia & $1.09 \pm 0.83$ & Roseburia & $2.98 \pm 2.02$ & Methyloversatilis & $1.33 \pm 1.45$ \\
\hline 8 & Halomonas & $1.07 \pm 0.71$ & Ruminococcus & $2.65 \pm 1.47$ & Succinivibrio & $1.22 \pm 0.20$ \\
\hline 9 & Pediococcus & $0.97 \pm 1.01$ & Clostridium & $2.56 \pm 0.42$ & Sutterella & $1.06 \pm 0.27$ \\
\hline 10 & Rhodococcus & $0.77 \pm 0.64$ & Helicobacter & $1.07 \pm 0.65$ & Salinispora & $1.01 \pm 0.37$ \\
\hline
\end{tabular}

Alpha diversity of the sequencing data and the analyses of intra- and inter-group difference in bacterial profile The rarefaction curves of number of observed species (Fig. 1a), Chao index (Fig. 1b), and Shannon index (Fig. 1c) reach a plateau, suggesting that the identified sequences may sufficiently cover the bacteria in the samples. The rank abundance curves (Fig. 1d) also become stable, indicating that species distribution is uniform. Both the Anosim and MRPP analyses revealed that the inter-group difference in bacterial profile was larger than the intra-group difference and the inter-group difference was statistically significant (All $P<0.01$, Table 3 ).

\section{Propionibacterium was specifically detected in patients with sarcoidosis}

We compared the relative abundance of each genus in the three patient groups. A total of 67 bacterial genera showed significantly higher or lower relative abundance in the sarcoidosis group compared with the TB and control groups $(P<0.01, \mathrm{q}<0.01)$. Among the 67 genera, 38 showed significantly lower relative abundance in the sarcoidosis group than in the TB and control groups $(P<0.01, \mathrm{q}<0.01$, Table 4$)$, and 29 showed significantly higher relative abundance in the sarcoidosis group than in the TB and control groups $(P<0.01, \mathrm{q}$ $<0.01$, Table 5).

Among the 29 bacterial genera with significantly higher relative abundance in the sarcoidosis group, The Propionibacterium Genus was found in all the 17 patients with sarcoidosis (100\% positive rate) but was completely absent in all the 8 patients with TB $(0 \%$ positive rate, $\left.X^{2}=25, P<0.0001\right)$ and 11 control patients $(0 \%$ positive rate, $X 2=28, P<0.0001)$. In contrast, the other 28 bacterial genera were detected in all the patients in the three groups, suggesting that these bacterial genera may not be specific for patients with sarcoidosis. The relative abundance of the Propionibacterium Genus in the sarcoidosis group was $0.16 \% \pm 0.11 \%$, which was significantly higher than that in the control $(0 \%$, $P=0.001, \mathrm{q}=0.0055)$ and TB groups $(0 \%, P=0.0010$, $q=0.0063)$.

In addition to propionibacteria, mycobacteria [7, 8, 27, 28], Borrelia [29, 30], mycoplasma [31], and chlamydia [32] have also been investigated as pathogenic organisms. Thus, we examined the four microorganisms. Mycobacterium was detected in the three patient groups but showed very low relative abundance. Although the relative abundance of Mycobacterium in the TB group $(0.077 \% \pm 0.037 \%)$ was higher than that in the sarcoidosis $(0.056 \% \pm 0.029 \%, P=0.18, q=0.38)$ and control $(0.052 \% \pm 0.073 \%, P=0.36, q=0.94)$ groups, the differences was not statistically significant. The relative abundance of mycoplasma was also very low in the three groups: $0.0067 \% \pm 0.0090 \%$ in the sarcoidosis group, $0.0026 \% \pm 0.0034 \%$ in the control group $(P=0.59$, $\mathrm{q}=0.77)$, and $0.0020 \% \pm 0.0023 \%$ in the TB group $(P$ $=0.19, \mathrm{q}=0.39$ ). The differences were not statistically significant. Neither Borrelia nor and chlamydia was detected in the samples.

The two predominant bacterial species in the Propionibacterium Genus are Propionibacterium acnes (P. acnes) and Propionibacterium granulosum (P. granulosum). P. acnes was detected in all the 17 patients with sarcoidosis, whereas P. granulosum was detected in only 5 of the 17 patients. Both Propionibacterium species were not found in the control and TB groups. The relative abundance of $\mathrm{P}$. acnes in the sarcoidosis group $(0.16 \% \pm 0.11 \%)$ was significantly higher than that in the control $(0 \%, P=0.0010, q=0.0038)$ and TB $(0 \%, P=$ $0.0010, \mathrm{q}=0.0044$ ) groups (Fig. $2 \mathrm{a}$ ). In contrast, the relative abundance of $\mathrm{P}$. granulosum in the sarcoidosis group $(0.0022 \% \pm 0.0044 \%)$, which was extremely lower than the relative abundance of P. acnes, was not significantly different compared with that in the control $(0 \%$, $P=0.088, \quad \mathrm{q}=0.18)$ and TB $(0 \%, \quad P=0.19, \quad \mathrm{q}=0.38)$ groups (Fig. 2b). These data indicate that $P$. acnes may 


\section{A}

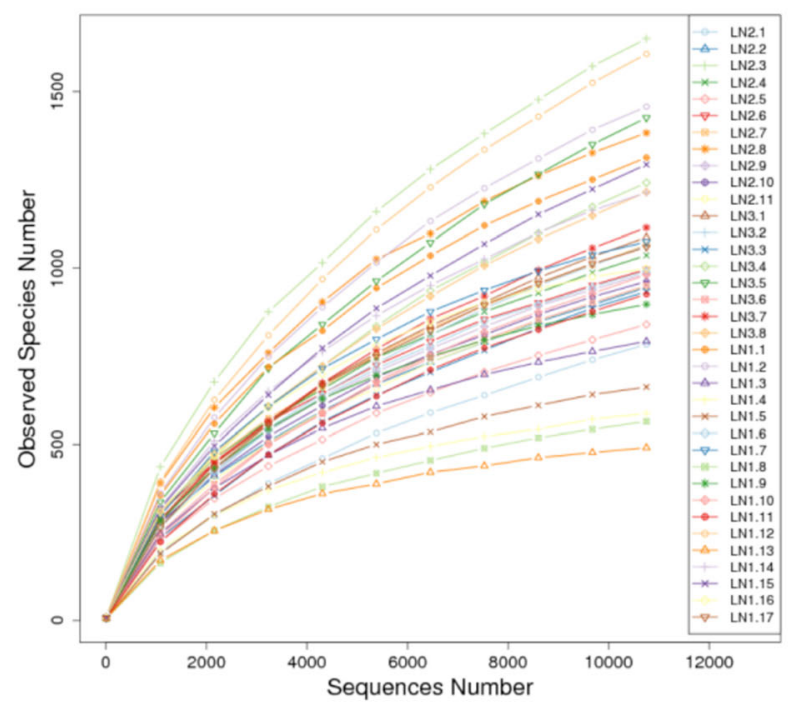

C

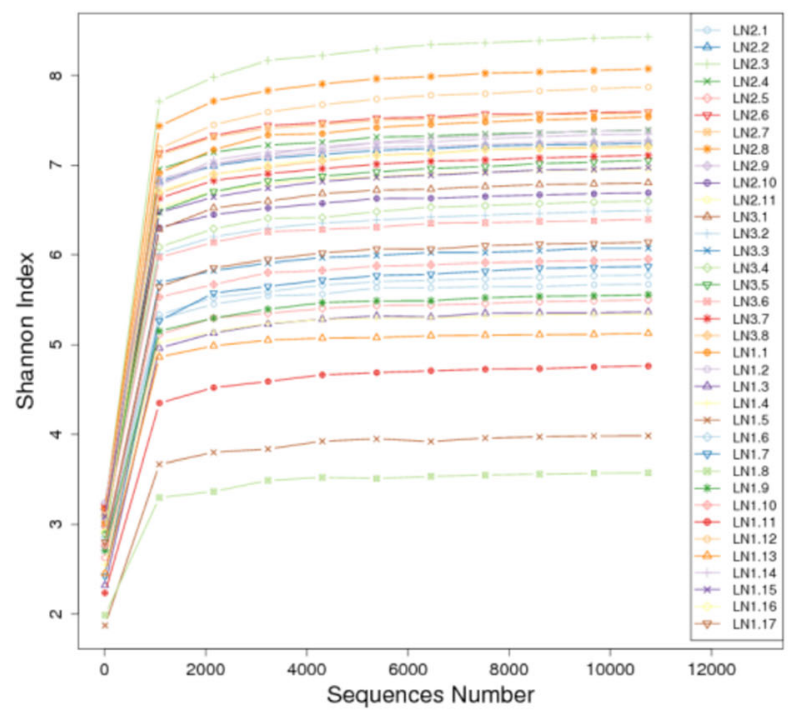

B

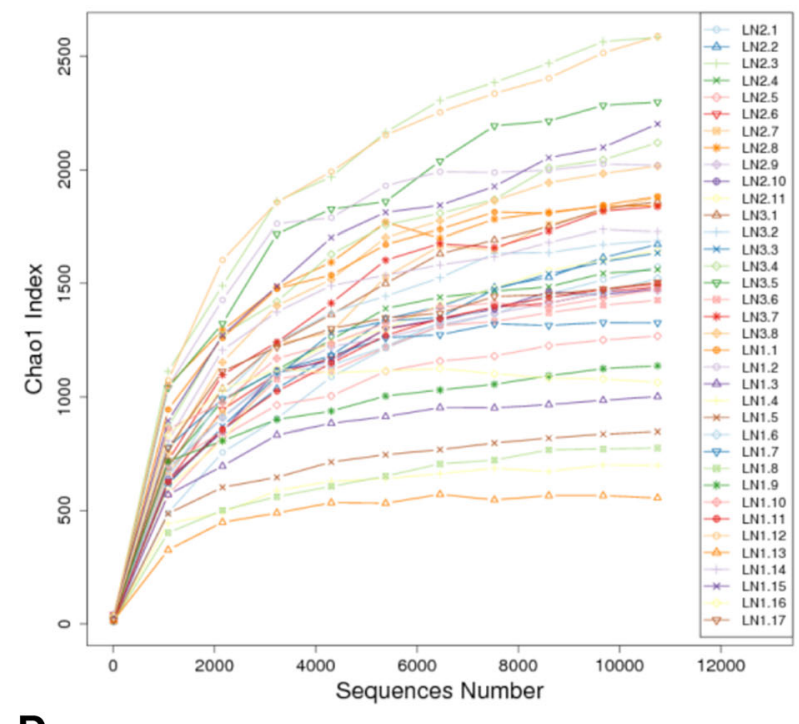

D

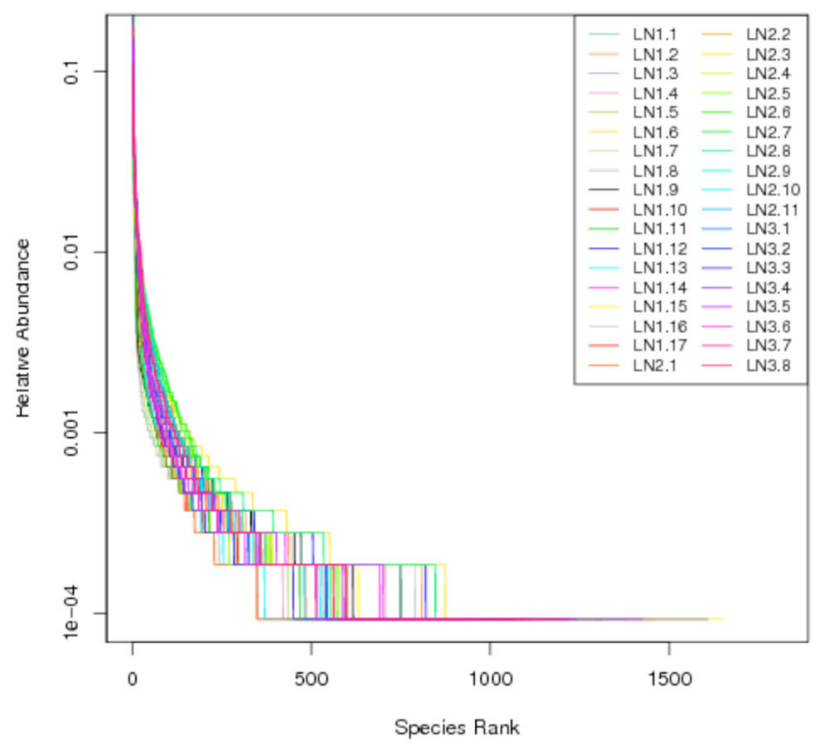

Fig. 1 Alpha diversity. a Number of observed species curves. b Chao index curves. c Shannon index curves. d Rank abundance curves. LN1: Sarcoidosis group. LN2: Control disease group. LN3: Tuberculosis group

Table 3 Inter-group analysis

\begin{tabular}{llllll}
\hline Comparing pair & \multicolumn{2}{l}{ Anosim analysis } & & MRPP analysis \\
\cline { 2 - 3 } & $R$ value & $P$ value & & A value & $P$ value \\
\hline Sarcoidosis versus Control & 0.9976 & 0.001 & & 0.2817 & 0.001 \\
Sarcoidosis versus Tuberculosis & 0.9957 & 0.001 & & 0.2772 & 0.001 \\
Tuberculosis versus Control & 0.4592 & 0.001 & & 0.09667 & 0.001 \\
\hline
\end{tabular}

be more likely to correlate with sarcoidosis than other bacteria. The process of identification of the unique bacterium in the sarcoidosis group is displayed in Fig. 3.

\section{Discussion}

The association of infection and sarcoidosis etiology has been studied extensively. Molecular biological approaches have been used to search bacterial genome in sarcoidosis lesions [33]. Previous studies have shown that possible pathogenic organisms for sarcoidosis include propionibacteria $[8,33,34]$, mycobacterium [7, 8, 27, 28], borrelia [29, 30], mycoplasma [31], 
Table 4 The 38 bacterial genera with significantly lower abundance in the sarcoidosis group

\begin{tabular}{|c|c|c|c|c|c|}
\hline \multirow[t]{2}{*}{ Bacteria Genera } & \multirow{2}{*}{$\begin{array}{l}\text { Sarcoidosis (\%) } \\
N=17\end{array}$} & \multirow{2}{*}{$\begin{array}{l}\text { Control (\%) } \\
N=11\end{array}$} & \multirow{2}{*}{$\begin{array}{l}\text { Tuberculosis (\%) } \\
N=8\end{array}$} & \multirow[t]{2}{*}{ q1 value } & \multirow[t]{2}{*}{ q2 value } \\
\hline & & & & & \\
\hline Prevotella & $0.26 \pm 0.37$ & $5.16 \pm 2.63$ & $2.75 \pm 1.29$ & $q<0.01$ & $q<0.01$ \\
\hline Clostridium & $0.16 \pm 0.14$ & $2.56 \pm 0.42$ & $2.60 \pm 0.25$ & $q<0.01$ & $q<0.01$ \\
\hline Parabacteroides & $0.018 \pm 0.27$ & $0.41 \pm 0.13$ & $0.25 \pm 0.066$ & $q<0.01$ & $q<0.01$ \\
\hline Bacteroides & $0.12 \pm 0.12$ & $13.13 \pm 2.72$ & $13.32 \pm 3.51$ & $q<0.01$ & $q<0.01$ \\
\hline Oscillospira & $0.12 \pm 0.085$ & $3.36 \pm 1.92$ & $0.58 \pm 0.31$ & $q<0.01$ & $q<0.01$ \\
\hline Methyloversatilis & $0.065 \pm 0.11$ & $0.28 \pm 0.15$ & $1.33 \pm 1.45$ & $q<0.01$ & $q<0.01$ \\
\hline Methylobacterium & $0.11 \pm 0.065$ & $4.11 \pm 3.95$ & $2.96 \pm 2.85$ & $q<0.01$ & $q<0.01$ \\
\hline Phascolarctobacterium & $0.069 \pm 0.063$ & $0.84 \pm 0.44$ & $0.59 \pm 0.20$ & $q<0.01$ & $q<0.01$ \\
\hline Roseburia & $0.055 \pm 0.069$ & $2.98 \pm 2.02$ & $0.71 \pm 0.19$ & $q<0.01$ & $q<0.01$ \\
\hline Faecalibacterium & $0.050 \pm 0.059$ & $0.58 \pm 0.15$ & $0.58 \pm 0.13$ & $q<0.01$ & $q<0.01$ \\
\hline Ruminococcus & $0.052 \pm 0.052$ & $0.52 \pm 0.14$ & $0.37 \pm 0.059$ & $q<0.01$ & $q<0.01$ \\
\hline Bradyrhizobium & $0.054 \pm 0.041$ & $0.27 \pm 0.093$ & $0.30 \pm 0.070$ & $q<0.01$ & $q<0.01$ \\
\hline Sutterella & $0.034 \pm 0.037$ & $0.87 \pm 0.31$ & $1.06 \pm 0.27$ & $q<0.01$ & $q<0.01$ \\
\hline Megamonas & $0.029 \pm 0.036$ & $0.54 \pm 0.20$ & $0.68 \pm 0.18$ & $q<0.01$ & $q<0.01$ \\
\hline Prevotella & $0.024 \pm 0.039$ & $3.16 \pm 1.02$ & $3.25 \pm 0.89$ & $q<0.01$ & $q<0.01$ \\
\hline Arcobacter & $0.018 \pm 0.022$ & $0.13 \pm 0.054$ & $0.41 \pm 0.47$ & $q<0.01$ & $q<0.01$ \\
\hline Dialister & $0.012 \pm 0.014$ & $0.076 \pm 0.019$ & $0.091 \pm 0.022$ & $q<0.01$ & $q<0.01$ \\
\hline Dorea & $0.0095 \pm 0.013$ & $0.086 \pm 0.057$ & $0.047 \pm 0.011$ & $q<0.01$ & $q<0.01$ \\
\hline Allobaculum & $0.0054 \pm 0.014$ & $0.36 \pm 0.35$ & $0.10 \pm 0.042$ & $q<0.01$ & $q<0.01$ \\
\hline Eubacterium & $0.0043 \pm 0.0089$ & $0.035 \pm 0.014$ & $0.039 \pm 0.012$ & $q<0.01$ & $q<0.01$ \\
\hline Acetobacter & $0.0059 \pm 0.0071$ & $0.025 \pm 0.011$ & $0.028 \pm 0.013$ & $q<0.01$ & $q<0.01$ \\
\hline Succinivibrio & $0.0045 \pm 0.0077$ & $0.70 \pm 0.30$ & $1.22 \pm 0.20$ & $q<0.01$ & $q<0.01$ \\
\hline Mitsuokella & $0.0032 \pm 0.0063$ & $0.032 \pm 0.012$ & $0.033 \pm 0.0096$ & $q<0.01$ & $q<0.01$ \\
\hline Anaerobiospirillum & $0.0031 \pm 0.0044$ & $0.10 \pm 0.037$ & $0.15 \pm 0.027$ & $q<0.01$ & $q<0.01$ \\
\hline Cetobacterium & $0.0033 \pm 0.0041$ & $0.077 \pm 0.077$ & $0.076 \pm 0.047$ & $q<0.01$ & $q<0.01$ \\
\hline Trichococcus & $0.0017 \pm 0.0045$ & $0.040 \pm 0.015$ & $0.074 \pm 0.050$ & $q<0.01$ & $q<0.01$ \\
\hline Flavisolibacter & $0.0022 \pm 0.0039$ & $0.022 \pm 0.029$ & $0.016 \pm 0.0084$ & $q<0.01$ & $q<0.01$ \\
\hline Collinsella & $0.0013 \pm 0.0039$ & $0.77 \pm 0.36$ & $0.79 \pm 0.18$ & $q<0.01$ & $q<0.01$ \\
\hline Brevibacillus & $0.0016 \pm 0.0032$ & $0.057 \pm 0.029$ & $0.082 \pm 0.046$ & $q<0.01$ & $q<0.01$ \\
\hline Peptococcus & $0.0012 \pm 0.0024$ & $0.052 \pm 0.017$ & $0.051 \pm 0.012$ & $q<0.01$ & $q<0.01$ \\
\hline Bilophila & $0.00065 \pm 0.0022$ & $0.011 \pm 0.0043$ & $0.010 \pm 0.0064$ & $q<0.01$ & $q<0.01$ \\
\hline Slackia & $0.00068 \pm 0.0020$ & $0.035 \pm 0.018$ & $0.042 \pm 0.018$ & $q<0.01$ & $q<0.01$ \\
\hline$J 2-29$ & $0.00065 \pm 0.0019$ & $5.28 \pm 3.82$ & $8.91 \pm 2.15$ & $q<0.01$ & $q<0.01$ \\
\hline Campylobacter & $0.00051 \pm 0.0016$ & $0.056 \pm 0.016$ & $0.071 \pm 0.024$ & $q<0.01$ & $q<0.01$ \\
\hline Thermicanus & $0.00026 \pm 0.001$ & $0.013 \pm 0.0071$ & $0.018 \pm 0.020$ & $q<0.01$ & $q<0.01$ \\
\hline Paraprevotella & $0 \pm 0$ & $0.13 \pm 0.13$ & $0.018 \pm 0.035$ & $q<0.01$ & $q<0.01$ \\
\hline Phormidium & $0 \pm 0$ & $0.011 \pm 0.018$ & $0.0089 \pm 0.012$ & $q<0.01$ & $q<0.01$ \\
\hline Sporolactobacillus & $0 \pm 0$ & $0.0093 \pm 0.019$ & $0.0074 \pm 0.0038$ & $q<0.01$ & $q<0.01$ \\
\hline
\end{tabular}

q1 value: Sarcoidosis versus Control q value. q2 value: Sarcoidosis versus Tuberculosis q value

and chlamydia [32]. Particularly, MTB and propionibacteria are the most frequently reported bacteria correlating with sarcoidosis $[35,36]$. In spite of the efforts, no consistent and definitive conclusion has been drawn. Piotrowski et al. reported that MTB infection triggered a sarcoid reaction, and thus they proposed that TB and sarcoidosis might be different clinical manifestations of MTB infection [28]. However, 
Table 5 The 29 bacterial genera with significantly higher abundance in the sarcoidosis group

\begin{tabular}{|c|c|c|c|c|c|}
\hline \multirow[t]{2}{*}{ Bacteria Genera } & \multirow{2}{*}{$\begin{array}{l}\text { Sarcoidosis (\%) } \\
N=17\end{array}$} & \multirow{2}{*}{$\begin{array}{l}\text { Control (\%) } \\
N=11\end{array}$} & \multirow{2}{*}{$\begin{array}{l}\text { Tuberculosis (\%) } \\
N=8\end{array}$} & \multirow[t]{2}{*}{ q1 value } & \multirow[t]{2}{*}{ q2 value } \\
\hline & & & & & \\
\hline Shewanella & $7.33 \pm 4.60$ & $0.014 \pm 0.015$ & $0.013 \pm 0.011$ & $q<0.01$ & $q<0.01$ \\
\hline Pseudomonas & $4.32 \pm 2.75$ & $0.12 \pm 0.052$ & $0.17 \pm 0.080$ & $q<0.01$ & $q<0.01$ \\
\hline Prochlorococcus & $2.24 \pm 2.83$ & $0.021 \pm 0.034$ & $0.090 \pm 0.096$ & $q<0.01$ & $q<0.01$ \\
\hline Paenibacillus & $0.59 \pm 2.14$ & $0.012 \pm 0.0095$ & $0.010 \pm 0.0069$ & $q<0.01$ & $q<0.01$ \\
\hline Pediococcus & $0.97 \pm 1.01$ & $0.088 \pm 0.095$ & $0.046 \pm 0.022$ & $q<0.01$ & $q<0.01$ \\
\hline Halomonas & $1.07 \pm 0.71$ & $0.0038 \pm 0.0041$ & $0.0053 \pm 0.0047$ & $q<0.01$ & $q<0.01$ \\
\hline Rhodococcus & $0.77 \pm 0.64$ & $0.012 \pm 0.0088$ & $0.011 \pm 0.0056$ & $q<0.01$ & $q<0.01$ \\
\hline Candidatus Portiera & $0.46 \pm 0.42$ & $0.0029 \pm 0.0032$ & $0.0059 \pm 0.0037$ & $q<0.01$ & $q<0.01$ \\
\hline Alteromonas & $0.34 \pm 0.30$ & $0.0032 \pm 0.0036$ & $0.0030 \pm 0.0052$ & $q<0.01$ & $q<0.01$ \\
\hline Idiomarina & $0.034 \pm 0.59$ & $0.00045 \pm 0.0010$ & $0.00033 \pm 0.00094$ & $q<0.01$ & $q<0.01$ \\
\hline Marinomonas & $0.30 \pm 0.27$ & $0.00020 \pm 0.00066$ & $0.00064 \pm 0.0012$ & $q<0.01$ & $q<0.01$ \\
\hline Vibrio & $0.21 \pm 0.24$ & $0.023 \pm 0.011$ & $0.033 \pm 0.015$ & $q<0.01$ & $q<0.01$ \\
\hline Corynebacterium & $0.25 \pm 0.15$ & $0.075 \pm 0.051$ & $0.052 \pm 0.011$ & $q<0.01$ & $q<0.01$ \\
\hline Tolumonas & $0.18 \pm 0.21$ & $0.0013 \pm 0.0019$ & $0.067 \pm 0.12$ & $q<0.01$ & $q<0.01$ \\
\hline HTCC & $0.19 \pm 0.17$ & $0.0047 \pm 0.0041$ & $0.016 \pm 0.024$ & $q<0.01$ & $q<0.01$ \\
\hline Glaciecola & $0.13 \pm 0.20$ & $0.0016 \pm 0.0021$ & $0.0011 \pm 0.0015$ & $q<0.01$ & $q<0.01$ \\
\hline Aliivibrio & $0.10 \pm 0.20$ & $0.0071 \pm 0.012$ & $0.0050 \pm 0.0043$ & $q<0.01$ & $q<0.01$ \\
\hline Swaminathania & $0.14 \pm 0.15$ & $0.00020 \pm 0.00066$ & $0.0011 \pm 0.0015$ & $q<0.01$ & $q<0.01$ \\
\hline Propionibacterium & $0.16 \pm 0.11$ & $0 \pm 0$ & $0 \pm 0$ & $q<0.01$ & $q<0.01$ \\
\hline Micrococcus & $0.10 \pm 0.16$ & $0.015 \pm 0.0071$ & $0.014 \pm 0.0071$ & $q<0.01$ & $q<0.01$ \\
\hline Thalassobius & $0.11 \pm 0.12$ & $0.013 \pm 0.010$ & $0.0073 \pm 0.0038$ & $q<0.01$ & $q<0.01$ \\
\hline Saccharopolyspora & $0.096 \pm 0.11$ & $0.0028 \pm 0.0040$ & $0.0045 \pm 0.012$ & $q<0.01$ & $q<0.01$ \\
\hline Ochrobactrum & $0.069 \pm 0.056$ & $0.0067 \pm 0.0053$ & $0.0097 \pm 0.011$ & $q<0.01$ & $q<0.01$ \\
\hline Weissella & $0.060 \pm 0.042$ & $0.0038 \pm 0.0046$ & $0.0033 \pm 0.0038$ & $q<0.01$ & $q<0.01$ \\
\hline MSBL3 & $0.050 \pm 0.051$ & $0.00021 \pm 0.00069$ & $0.00063 \pm 0.0012$ & $q<0.01$ & $q<0.01$ \\
\hline SGSH944 & $0.039 \pm 0.052$ & $0.0013 \pm 0.0041$ & $0.00033 \pm 0.00093$ & $q<0.01$ & $q<0.01$ \\
\hline Methylophaga & $0.032 \pm 0.046$ & $0.0025 \pm 0.0040$ & $0.0019 \pm 0.0018$ & $q<0.01$ & $q<0.01$ \\
\hline Thauera & $0.029 \pm 0.048$ & $0.0011 \pm 0.0022$ & $0.0033 \pm 0.0052$ & $q<0.01$ & $q<0.01$ \\
\hline Xanthobacter & $0.015 \pm 0.013$ & $0.00045 \pm 0.00099$ & $0.0013 \pm 0.0020$ & $q<0.01$ & $q<0.01$ \\
\hline
\end{tabular}

q1 value: Sarcoidosis versus Control q value. q2 value: Sarcoidosis versus Tuberculosis q value

our previous study, which determined the copy number of MTB in lymph node specimens of patients with TB or sarcoidosis, suggests that MTB may not be the pathogenic bacterium of sarcoidosis [8].

Propionibacteria are gram positive anaerobic bacteria and belong to the normal flora of the skin. Since Abe et al. first isolated P. acnes from lymph node biopsy specimens of patients with sarcoidosis in 1984 [34], P. acnes has been suspected to be a pathogenic bacterium for sarcoidosis. Negi et al. used P. acnes-specific monoclonal antibodies that react with cell-membrane-bound lipoteichoic acid (PAB antibody) in immunohistochemistry to locate P. acnes in the lung tissue and lymph node specimens of patients with sarcoidosis, and they discovered that the PAB antibodies specifically bound to the sarcoidosis specimens but did not react with tissues from patients with non-sarcoidosis disease [33]. We previously performed a meta-analysis to systematically review the articles that focused on the association of $\mathrm{P}$. acnes and sarcoidosis [37], and our results support that P. acnes is highly likely a pathogen of sarcoidosis.

On the other hand, other studies indicate that P. acnes may not be associated with sarcoidosis. Ishige et al. found that $56 \%-73 \%$ of specimens (lung tissues: $24 / 43$, mediastinal lymph node specimens: $8 / 11$ ) from patients with non-sarcoidosis diseases showed positive P. acnes culture [38]. Oswald et al. discovered that compared with the CD4+ and/or CD8+ T cells from the controls, the $\mathrm{T}$ cells from patients with sarcoidosis reacted to mycobacterial ESAT-6 protein more frequently but 


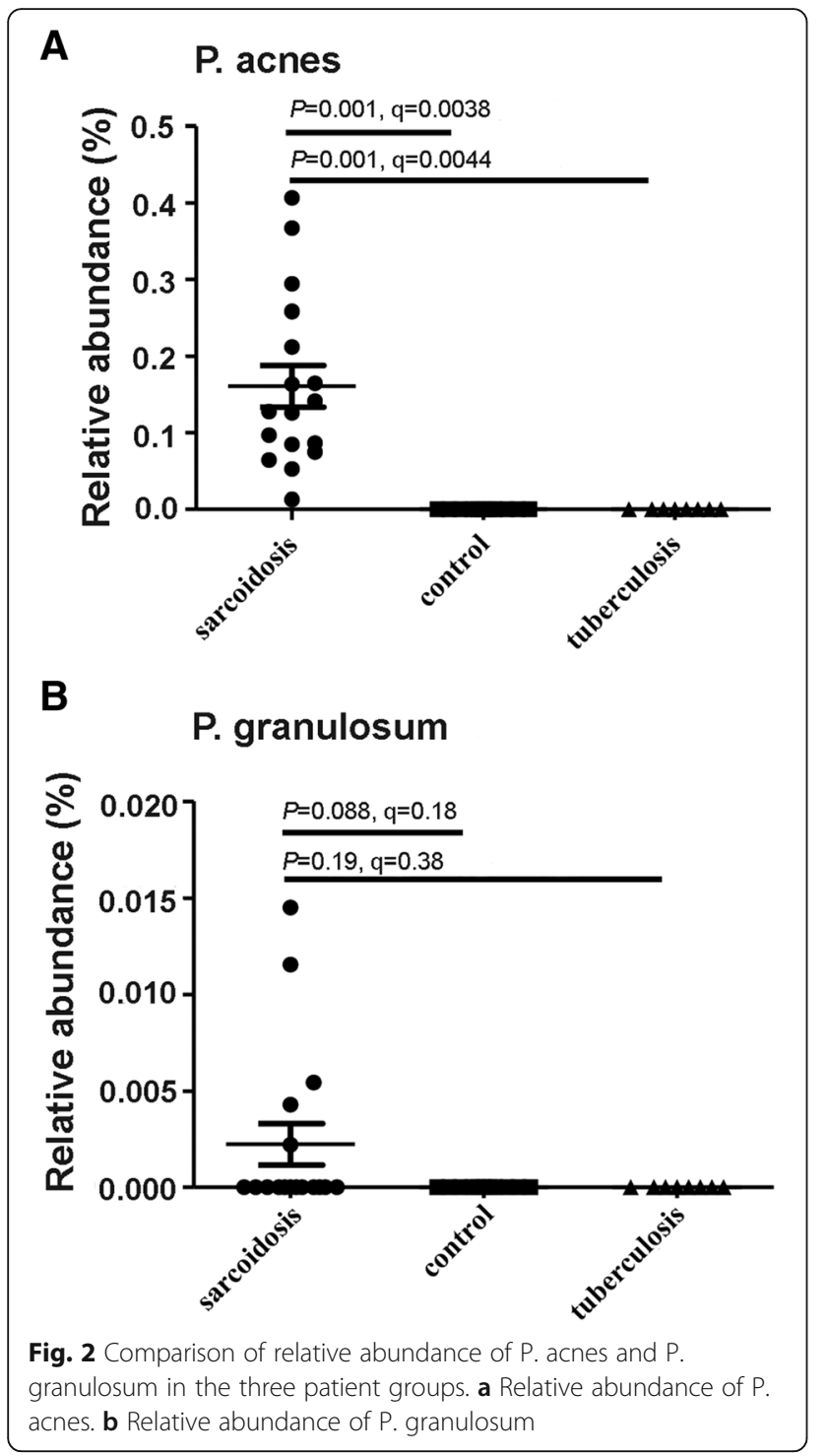

showed similar response to P. acnes proteins [7]. They also used matrix-assisted laser desorption ionization imaging mass spectrometry to locate the ESAT- 6 protein and the $\mathrm{P}$. acnes proteins in the tissue specimens and found that ESAT-6 signals specifically existed in sarcoidosis granulomas, whereas P. acnes signals were distributed non-specially in the tissues [7]. These studies suggest that P. acnes may be not involved in sarcoidosis etiology.

To identify potential pathogenic bacteria of sarcoidosis, we previously used quantitative PCR to quantify the copy number of 16SrRNA gene of P. acnes and P. granulosum in the lymph node specimens and found that propionibacteria existed in $80 \%$ of sarcoidosis specimens but only in $4.5 \%$ of TB specimens [9]. To further confirm our previous conclusions, in the current study, we used high throughput 16SrRNA gene sequencing to

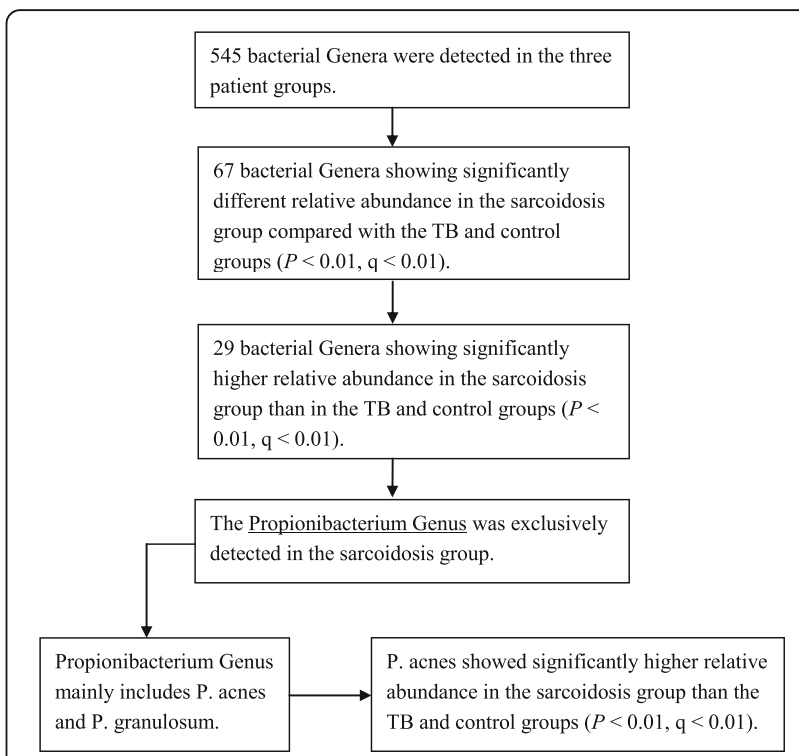

Fig. 3 Identification of the unique bacteria in the sarcoidosis group

investigate the bacterial profile of lymph node specimens of sarcoidosis, TB, or other lung diseases. Our results showed that $\mathrm{P}$. acnes were exclusively present in sarcoidosis samples, and both P. acnes and P. granulosum were not detected in the TB and control groups. These findings clearly support that P. acnes is associated with sarcoidosis.

The possible pathogenic mechanism of $\mathrm{P}$. acnes in sarcoidosis development has been tested in animal models. Kouji Iio et al. used heat-inactivated P. acnes and Freund's complete adjuvant to induce pulmonary granuloma in mice, and found that the total number of lymphocytes and the ratio of $\mathrm{CD} 4+$ to $\mathrm{CD} 8+\mathrm{T}$ cells in the bronchoalveolar lavage fluid of the mice with pulmonary granuloma were higher than those of the control mice [39]. Their study suggests that P. acnes may cause pulmonary allergic inflammation, which may consequently lead to granuloma. Eishi et al. also proposed that P. acnes could cause sarcoidosis by an allergic endogenous infection [40]. P. acnes or the bacteria-related antigens may interact with the pattern recognition receptors to stimulate macrophages and $\mathrm{T}$ cells to release pro-inflammatory factors, such as IFN- $\gamma$, IL-2, TNF- $\alpha$, and IL-6, which ultimately disrupts the immune homeostasis in patients and induces sarcoidosis development [36]. Thus, P. acnes may cause sarcoidosis by interfering in the immune homeostasis.

Compared with quantitative PCR approach, high throughput16SrRNA gene sequencing is a more effective approach to investigate bacterial profile of biopsy specimens and identify the predominant bacterial species in the specimens. The sequencing approach also allows us 
to compare the diversity and relative abundance of bacteria in specimens. Although quantitative PCR approach may be sensitive, low-cost, and fast, the sequencing approach can produce data for comprehensive analyses, such as bacterial taxonomy, bacterial diversity, and the correlation of bacterial profile evolution and sarcoidosis progression. The current study first used high throughput sequencing approach to investigate the bacterial profile in lymph node specimens of patients with sarcoidosis and to identify that propionibacteria were exclusively present in the sarcoidosis specimens. The advantages of the sequencing method include high throughput, high reproducibility, and high accuracy. The limitations of the sequencing method include technically complex and requiring special instrument and software for the analysis. In addition, the roles of those bacteria with significantly higher or lower abundance in the sarcoidosis group compared with the control and TB groups in the development of sarcoidosis remain unclear. Furthermore, the control groups in the current study included lymph node specimens of patients with nonspecific lymphadenitis and of patients with mediastinal lymph node metastasis of lung cancer, which may contribute to the highly various bacterial profiles in the control group. Another limitation of the current study is that the number of tissue specimens in the current study was relatively small. We are currently collecting more samples to further investigate the dynamic evolution of bacterial profiles in the sarcoidosis lesions at different disease stages.

\section{Conclusions}

The current study used high throughput 16SrRNA gene sequencing to demonstrate that $\mathrm{P}$. acnes exclusively existed in the mediastinal lymph node specimens of patients with sarcoidosis but was absence in the specimens of patients with $\mathrm{TB}$ or other non-infectious lung diseases. These findings indicate that $\mathrm{P}$. acnes may correlate with sarcoidosis etiology.

\section{Additional files}

Additional file 1: Table S1. Sequencing data of the 36 tissue speciments. (XLS $15 \mathrm{~kb})$

Additional file 2: Table S2. Sequences of the unique OTUs. (XLS $3616 \mathrm{~kb}$ ) Additional file 3: Table S3. Taxonomic annotation of the unique OTUs. (XLS $1114 \mathrm{~kb}$ )

Additional file 4: Table S4. Relative abundance of the 545 bacterial genera in each sample. (XLS $330 \mathrm{~kb}$ )

\section{Abbreviations}

Anosim: The analysis of similarity; MRPP: Multi-response permutations procedure; MTB: Mycobacterium tuberculosis; OTU: Operational taxonomic unit; P. acnes: Propionibacterium acnes; P. granulosum: Propionibacterium granulosum; PCR: Polymerase chain reaction.; QIIME: Quantitative insights into microbial ecology; TB: Tuberculosis

\section{Acknowledgments}

This study was funded by grants from the National Science Foundation of China (No: 81670055,81670056,91442103, 81500052 and 81570057), Ministry of Science and Technology of the People's Republic of China (2016YFC1100200, 2016YFC1100204), the Health Bureau Program of Shanghai Municipality (SHDC12014120 and 2013SY047), and Tongji University (1511219020). The authors thank Professor Robert P.

Baughman for proofreading the manuscript.

\section{Funding}

This study was funded by grants from the National Science Foundation of China (No: 81670055,81670056,91442103, 81500052 and 81570057), Ministry of Science and Technology of the People's Republic of China (2016YFC1100200, 2016YFC1100204), the Health Bureau Program of Shanghai Municipality (SHDC12014120 and 2013SY047), and Tongji University (1511219020).

\section{Availability of data and material}

Bacterial $16 \mathrm{~S}$ ribosomal RNA gene sequences were collected and analyzed to annotate taxonomic information using the GreenGene database.

\section{Authors' Contributions}

HPL, DW, MMZ, SSD, QHL and TC participated in the conception, hypothesis, and design of the study. MMZ, SSD and QHL performed the experiments. MMZ, DW and TC carried out the statistical analyses. All authors contributed to interpretation of the data. MMZ and HPL wrote the manuscript and all authors made critical revisions. All authors read and approved the final manuscript.

\section{Competing financial interests}

The authors declare that there are no competing financial interests.

\section{Consent for publication}

We have obtained consent to publish from all the participants.

\section{Ethics approval and consent to participate}

The protocol for collecting and handling patients' biopsy specimens has been approved by the Institutional Review Board of Shanghai Pulmonary Hospital and Tongji University School of Medicine (Approval No: 2011-FK-10). All the participating patients signed the informed consent.

Received: 21 September 2016 Accepted: 19 January 2017

Published online: 01 February 2017

\section{References}

1. Costabel U, Hunninghake GW. ATS/ERS/WASOG statement on sarcoidosis. Sarcoidosis statement committee. American thoracic society. European respiratory society. World association for sarcoidosis and other granulomatous disorders. Eur Respir J. 1999;14(4):735-7.

2. Celada $L$, Hawkins C, Drake WP. The etiologic role of infectious antigens in sarcoidosis pathogenesis. Clin Chest Med. 2015;36(4):561-8.

3. Fingerlin TE, Hamzeh N, Maier LA. Genetics of sarcoidosis. Clin Chest Med. 2015:36(4):569-84.

4. Zissel G, Muller-Quernheim J. Cellular players in the immunopathogenesis of sarcoidosis. Clin Chest Med. 2015;36(4):549-60.

5. Newman LS, Rose CS, Bresnitz EA, Rossman MD, Barnard J, Frederick M, Terrin ML, Weinberger SE, Moller DR, McLennan G, Hunninghake G, DePalo L, Baughman RP, lannuzzi MC, Judson MA, Knatterud GL, Thompson BW, Teirstein AS, Yeager Jr H, Johns CJ, Rabin DL, Rybicki BA, Cherniack R. A case control etiologic study of sarcoidosis: environmental and occupational risk factors. Am J Respir Crit Care Med. 2004;170(12):1324-30.

6. Eishi Y, Suga M, Ishige I, Kobayashi D, Yamada T, Takemura T, Takizawa T, Koike M, Kudoh S, Costabel U, Guzman J, Rizzato G, Gambacorta M, du Bois R, Nicholson AG, Sharma OP, Ando M. Quantitative analysis of mycobacterial and propionibacterial DNA in lymph nodes of Japanese and European patients with sarcoidosis. J Clin Microbiol. 2002;40(1):198-204.

7. Oswald-Richter KA, Beachboard DC, Seeley EH, Abraham S, Shepherd BE, Jenkins CA, Culver DA, Caprioli RM, Drake WP. Dual analysis for mycobacteria and propionibacteria in sarcoidosis BAL. J Clin Immunol. 2012;32(5):1129-40.

8. Zhou Y, Li HP, Li QH, Zheng H, Zhang RX, Chen G, Baughman RP. Differentiation of sarcoidosis from tuberculosis using real-time PCR assay for 
the detection and quantification of mycobacterium tuberculosis. Sarcoidosis Vasc Diffuse Lung Dis. 2008;25(2):93-9.

9. Zhou Y, Wei YR, Zhang Y, Du SS, Baughman RP, Li HP. Real-time quantitative reverse transcription-polymerase chain reaction to detect propionibacterial ribosomal RNA in the lymph nodes of Chinese patients with sarcoidosis. Clin Exp Immunol. 2015;181(3):511-7.

10. Caporaso JG, Lauber CL, Walters WA, Berg-Lyons D, Lozupone CA, Turnbaugh PJ, Fierer N, Knight R. Global patterns of 16S rRNA diversity at a depth of millions of sequences per sample. Proc Natl Acad Sci U S A. 2011;108 Suppl 1:4516-22.

11. Hess M, Sczyrba A, Egan R, Kim TW, Chokhawala H, Schroth G, Luo S, Clark DS, Chen F, Zhang T, Mackie RI, Pennacchio LA, Tringe SG, Visel A, Woyke T, Wang Z, Rubin EM. Metagenomic discovery of biomass-degrading genes and genomes from cow rumen. Science. 2011;331(6016):463-7.

12. Degnan $\mathrm{PH}, \mathrm{Ochman} \mathrm{H}$. Illumina-based analysis of microbial community diversity. Isme j. 2012;6(1):183-94.

13. Youssef N, Sheik CS, Krumholz LR, Najar FZ, Roe BA, Elshahed MS. Comparison of species richness estimates obtained using nearly complete fragments and simulated pyrosequencing-generated fragments in 16S rRNA gene-based environmental surveys. Appl Environ Microbiol. 2009;75(16):5227-36.

14. Li QH, Li HP, Shen YP, Zhao L, Shen L, Zhang Y, Jiang DH, Baughman RP. A novel multi-parameter scoring system for distinguishing sarcoidosis from sputum negative tuberculosis. Sarcoidosis Vasc Diffuse Lung Dis. 2012;29(1):11-8,

15. Medical Association Chinese Society for Tuberculosis,Chinese. Tuberculosis diagnosis and treatment guidelines. Chinese Practical J Rural doctor (in Chinese). 2013;02(20):7-11.

16. Magoc T, Salzberg SL. FLASH: fast length adjustment of short reads to improve genome assemblies. Bioinformatics. 2011;27(21):2957-63.

17. Edgar RC, Haas BJ, Clemente JC, Quince C, Knight R. UCHIME improves sensitivity and speed of chimera detection. Bioinformatics. 2011;27(16):2194-200.

18. Haas BJ, Gevers D, Earl AM, Feldgarden M, Ward DV, Giannoukos G, Ciulla D, Tabbaa D, Highlander SK, Sodergren E, Methe B, DeSantis TZ, Petrosino JF, Knight R, Birren BW. Chimeric 16S rRNA sequence formation and detection in Sanger and 454-pyrosequenced PCR amplicons. Genome Res. 2011; 21(3):494-504.

19. Edgar RC. UPARSE: highly accurate OTU sequences from microbial amplicon reads. Nat Methods. 2013;10(10):996-8.

20. DeSantis TZ, Hugenholtz P, Larsen N, Rojas M, Brodie EL, Keller K, Huber T, Dalevi D, Hu P, Andersen GL. Greengenes, a chimera-checked 165 rRNA gene database and workbench compatible with ARB. Appl Environ Microbiol. 2006;72(7):5069-72.

21. Wang Q, Garrity GM, Tiedje JM, Cole JR. Naive Bayesian classifier for rapid assignment of rRNA sequences into the new bacterial taxonomy. Appl Environ Microbiol. 2007;73(16):5261-7.

22. Caporaso JG, Kuczynski J, Stombaugh J, Bittinger K, Bushman FD, Costello EK, Fierer N, Pena AG, Goodrich JK, Gordon JI, Huttley GA, Kelley ST, Knights D, Koenig JE, Ley RE, Lozupone CA, McDonald D, Muegge BD, Pirrung M, Reeder J, Sevinsky JR, Turnbaugh PJ, Walters WA, Widmann J, Yatsunenko T, Zaneveld J, Knight R. QIIME allows analysis of high-throughput community sequencing data. Nat Methods. 2010;7(5):335-6.

23. White JR, Nagarajan N, Pop M. Statistical methods for detecting differentially abundant features in clinical metagenomic samples. PLoS Comput Biol. 2009;5(4):e1000352.

24. Cai L. Multi-response permutation procedure as an alternative to the analysis of variance: an SPSS implementation. Behav Res Methods. 2006;38(1):51-9.

25. Clarke KR. Non-parametric multivariate analyses of changes in community structure. Aust J Ecol. 1993;18:117-43.

26. Storey JD, Tibshirani R. Statistical significance for genomewide studies. Proc Natl Acad Sci U S A. 2003;100(16):9440-5.

27. Oswald-Richter KA, Beachboard DC, Zhan X, Gaskill CF, Abraham S, Jenkins C, Culver DA, Drake W. Multiple mycobacterial antigens are targets of the adaptive immune response in pulmonary sarcoidosis. Respir Res. 2010;11:161.

28. Piotrowski WJ, Gorski P, Duda-Szymanska J, Kwiatkowska S. Mycobacterium tuberculosis as a sarcoid factor? a case report of family sarcoidosis. Am J Case Rep. 2014;15:216-20.

29. Jacob F. Could Borrelia burgdorferi be a causal agent of sarcoidosis? Med Hypotheses. 1989;30(4):241-3.

30. Derler AM, Eisendle K, Baltaci M, Obermoser G, Zelger B. High prevalence of 'Borrelia-like' organisms in skin biopsies of sarcoidosis patients from Western Austria. J Cutan Pathol. 2009;36(12):1262-8.
31. Johnson LA, Edsall JR, Austin JH, Ellis K. Pulmonary sarcoidosis: could mycoplasma-like organisms be a cause? Sarcoidosis Vasc Diffuse Lung Dis. 1996;13(1):38-42.

32. Puolakkainen M, Campbell LA, Kuo CC, Leinonen M, Gronhagen-Riska C, Saikku P. Serological response to Chlamydia pneumoniae in patients with sarcoidosis. J Infect. 1996;33(3):199-205.

33. Negi M, Takemura T, Guzman J, Uchida K, Furukawa A, Suzuki Y, lida T, Ishige I, Minami J, Yamada T, Kawachi H, Costabel U, Eishi Y. Localization of propionibacterium acnes in granulomas supports a possible etiologic link between sarcoidosis and the bacterium. Mod Pathol. 2012;25(9):1284-97.

34. Abe C, Iwai K, Mikami R, Hosoda Y. Frequent isolation of Propionibacterium acnes from sarcoidosis lymph nodes. Zentralbl Bakteriol Mikrobiol Hyg A. 1984;256(4):541-7.

35. Chen ES, Wahlstrom J, Song Z, Willett MH, Wiken M, Yung RC, West EE, McDyer JF, Zhang Y, Eklund A, Grunewald J, Moller DR. T cell responses to mycobacterial catalase-peroxidase profile a pathogenic antigen in systemic sarcoidosis. J Immunol. 2008;181(12):8784-96.

36. Furusawa H, Suzuki Y, Miyazaki Y, Inase N, Eishi Y. Th1 and Th17 immune responses to viable Propionibacterium acnes in patients with sarcoidosis. Respir Investig. 2012;50(3):104-9.

37. Zhou Y, Hu Y, Li H. Role of Propionibacterium acnes in sarcoidosis: a meta-analysis. Sarcoidosis Vasc Diffuse Lung Dis. 2013;30(4):262-7.

38. Ishige I, Eishi Y, Takemura T, Kobayashi I, Nakata K, Tanaka I, Nagaoka S, Iwai $\mathrm{K}$, Watanabe K, Takizawa T, Koike M. Propionibacterium acnes is the most common bacterium commensal in peripheral lung tissue and mediastinal lymph nodes from subjects without sarcoidosis. Sarcoidosis Vasc Diffuse Lung Dis. 2005;22(1):33-42.

39. lio K, lio TU, Okui Y, Ichikawa H, Tanimoto Y, Miyahara N, Kanehiro A, Tanimoto M, Nakata Y, Kataoka M. Experimental pulmonary granuloma mimicking sarcoidosis induced by Propionibacterium acnes in mice. Acta Med Okayama. 2010;64(2):75-83.

40. Eishi Y. Etiologic aspect of sarcoidosis as an allergic endogenous infection caused by Propionibacterium acnes. Biomed Res Int. 2013;2013:935289.

\section{Submit your next manuscript to BioMed Central and we will help you at every step:}

- We accept pre-submission inquiries

- Our selector tool helps you to find the most relevant journal

- We provide round the clock customer support

- Convenient online submission

- Thorough peer review

- Inclusion in PubMed and all major indexing services

- Maximum visibility for your research

Submit your manuscript at www.biomedcentral.com/submit
Biomed Central 